\title{
Nils Runeby
}

\section{Notizen über eine verhinderte Republik}

Um die Mitte des 17. Jahrhunderts, zur Zeit Königin Christines, kam es in Schweden zu heftigen Auseinandersetzungen zwischen dem Adelsstand und den nichtadeligen Stānden. Die Abalienationspolitik, das Verhāltnis zwischen König und Ständen und der Stände zueinander wurde in Frage gestellt; die Situation spitzte sich dramatisch zu, weil auch die Sukzessionsfrage und die Stellung des Königs in die Debatte hineingezogen wurden ${ }^{1}$. In einem bekannten und in der Literatur oft erwähnten und kommentierten Pamphlet von adeliger Tendenz klagten sich die Sprecher der vier Stände gegenseitig an, das richtige Gleichgewicht im Reiche zu gefährden. Hier wird dem Adel vorgeworfen, entweder „so die Zügel der Könige selbst zu greifen und ihre potentiam zu moderieren, daß ein König nicht mehr herrsche, als der Adel will“, oder „eine Aristocratia oder irgendwelche andere Form von Republique formieren zu wollen“, wodurch auch die anderen Stände unterdrückt würden.

Der Adelige antwortet indigniert, daß der Adel die vornehmste Stütze des Königs sei, daß bei einer Schwächung des Adelsstandes das größte „Fundament auf [ein] populares imperium und [eine] Veränderung des Staates" gelegt werde, und er klagt vor allem die Bürger und Geistlichen an: „Sie sind treffliche Respublicaner: ich denke Sie träumen, dass Sie in Rom sind, dass die Bürgermeister Marius und Cinna noch regieren, oder Sie haben so lange in Holland studieret und dort das popularische Regiment eingesauget, dass Sie denken [daß] eine Monarchie nicht mehr existiere“. Durch ihr Benehmen auf dem Reichstag und ihre Forderungen auf ein ständisches Initiativrecht und auf gemeinsame Beratungen „bringen Sie eine Menge Novitäten zur Sprache und greifen in arcana status“. Die Königin werde sie so strafen, daß sie ein anderes Mal „jura majestatis und regalia unturbieren lassen“. Die Bürgermeister werden außerdem beschuldigt, nicht ihren eigentlichen Pflichten nachzugehen, sondern sich mehr dafür zu interessieren, wie es bei Hofe zugehe, französisch und italienisch zu sprechen und Tacitus zu zitieren. In gleicher Weise wird gegen die Geistlichen der Vorwurf erhoben, Machiavelli, Campanella und Clapmarius statt der Bibel zu studieren.

\footnotetext{
${ }^{1} \mathrm{Vgl}$. Michael Roberts, Queen Christina and the general crisis of the seventeenth century, in: Past and present (1962). Die zitierten Pamphlete sind in Nils Runeby, Monarchia mixta (Studia historica upsaliensia 6, Stockholm 1962) erwähnt worden.
} 
Man könnte mit einigem Recht behaupten, daß die Beschuldigungen gegen die nichtadeligen Stände zu damaliger Zeit in Schweden verhältnismäßig neu waren, und daß man jetzt bei diesen Ständen tatsächlich neue politische Ansprüche (und Lesefrüchte) finden konnte. Adelsfeindliche Aussprüche oder Versuche, die adeligen Bestrebungen verdächtig zu machen, können hingegen schon früher ohne Schwierigkeiten nachgewiesen werden. Während der Vormundschaft der Königin Christine heißt es in einer auf Deutsch geschriebenen Darstellung: „trug der gantze stat die gestalt einer aristocratij“. Es gebe keinen Grund, heißt es in einer anderen Schrift, aus Schweden einen dänischen oder polnischen Staat zu machen. Seit Gustav Vasa haben nāmlich die Könige in Schweden „eine angemessene Macht und die Stände haben einander im Gleichgewicht gehalten“. Schon Gustav Adolf selbst klagte - in einem Entwurf zu einer Geschichte seiner Zeit aus den 1620er Jahren - die adelige Generation am Ende des 16. Jahrhunderts an, umstürzlerische Ambitionen zu hegen. Sie wolle „die Gehorsamkeit des Volkes von den Königen abwenden, um dann mit der Zeit in aller Ruhe ihre eigene Macht befestigen“ zu können. Gustav Adolfs heftige Worte, schreibt Sven Lundkvist in einem Kommentar zu eben diesem Zitat, zeigen "seine Angst vor einer Adelsrepublik wider die Fundamentalgesetze Schwedens und das schwedische Recht". Das Regiment des Reichsrates und des Hochadels würde nach Auffassung des Königs das Reich ins Chaos stürzen, und nur die Königsmacht könne die rettende und einende Kraft sein. Für den einflußreichen Mitarbeiter des Königs, Axel Oxenstierna, dagegen „gab es immer die Adelsrepublik als Ideal im Hintergrund“2.

$\mathrm{Ob}$ dies nun wirklich die Idealvorstellungen des Reichskanzlers waren, ist eine umstrittene Frage. Offiziell (und belehrend) erklärt Axel Oxenstierna in seinen Gesprächen mit Bulstrode Whitelocke, wie das richtige schwedische System funktioniere: Alle im Reich kennen ihre Rechte und Pflichten, das Volk nimmt an den Reichstagen teil, die Macht des Königs hat bestimmte Grenzen und „both the king and people are content with what they have and indeavour nothing of disquiet unto either" ${ }^{\text {. }}$.

Wenn ein Stand versuche, eine Vormachtstellung zu gewinnen, werde - nach den Pamphletschreibern - die notwendige Einheit des Reiches gestört. In Polen und Dänemark (vor 1660) herrsche eigentlich die Aristokratie, in Holland gebe es (nach 1650) das „popularische Regiment“. In Schweden dagegen mit seiner ,bedingten“ Erbmonarchie, mit Reichsrat und Vierständereichstag sei die Staatsform richtig, weil hier ein angemessenes Gleichgewicht zwischen König und Ständen bestehe. Mit einer gewissen Skepsis wurden auch Veränderungen in der Nachbarschaft zur Kenntnis genommen.

${ }^{2}$ Sven Lundkvist, Verklighetsuppfattning och verklighet. En studie i Gustav II Adolfs handlingsramar, in: Studier i äldre historia tillägnade Herman Schück (Stockholm 1985). Unter anderem Gesichtspunkt kann Günter Barudio in seiner großen Biographie: Gustav Adolf - der Große. Eine politische Biographie (Frankfurt/Main 1982) von einer „Monarchie im libertāren und republikanischen Sinne“ und von dem "Republikanismus seines / = Gustav Adolfs / Königtums“ $(90,351)$ sprechen. Zur Forschungslage siehe den zusammenfassenden Artikel von Erling Ladewig Petersen, Gustav II Adolf. En oversigt over nyere forskning, in: Historisk tidsskrift (Dänemark) 83 (1983).

${ }^{3}$ Vgl. Gerald E. Aylmer, English Perceptions of Scandinavia in the 17th Century, in: Europe and Scandinavia. Aspects of the Process of Integration in the 17th Century, hrsg. von Göran Rystad (Lund Studies in International History 18, Lund 1983). 
In einem Bericht über die neuesten Nachrichten aus Kopenhagen 1660 fragt sich ein schwedischer Diplomat, was nun mit den Gesetzen des Landes und den ,juribus ordinum regni“ passiere, wenn der König absolut geworden sei, und ob da nicht „Confusion" und Durcheinander entstünden. Und was sei denn eigentlich mit dem Begriff ,absolut' gemeint?

Wie erwähnt, konnte die schwedische ,Mischung' unterschiedlich interpretiert und politisch ausgenutzt werden ${ }^{4}$, und man kann beobachten, daß auf dem Weg von einer ständischen Verteidigung gewisser herkömmlicher Rechte und Vorstellungen von einer gemischten Staatsform bis hin zu einem aristokratischen oder, popularischen“ Regiment offenbar verschiedene ,Grauzonen“ existierten, die unklar erscheinen können, die aber in einem ,republikanischen' Zusammenhang nicht unwichtig sind. Diese Unklarheiten werden in den Beiträgen des Colloquiums mehrmals betont ${ }^{5}$, wobei auch die Notwendigkeit hervorgehoben wurde, den Republikbegriff zu problematisieren, der in seiner ,richtigen' Form nur bei Spinoza vorhanden gewesen zu sein scheint.

In diesem Zusammenhang kann es auch sinnvoll sein, Schweden heranzuziehen, obwohl hier weder im 16. noch im 17. Jahrhundert eine, Republik zustande kam. Beachtet man aber die eigenartigen,Grauzonen' und die politische Praxis, werden bereits die Bürgerkriege der späten Unionszeit und des 16. Jahrhunderts von Interesse sowie die häufigen Thronwechsel, die Minorennitätsperioden und Vormundschaftsregierungen des 17. Jahrunderts. Im 18. Jahrhundert kam Schweden an eine Republik so nahe wie nur möglich heran - nur der Name fehlte -, und sehr frühe Ansätze zu einem Parteiensystem liegen vor ${ }^{6}$. Hier muß nicht nur die Frage gestellt werden, wie es nach dem Tode Karls XII. dazu kam, sondern auch, welche Voraussetzungen und Traditionen vorhanden waren, die dorthin führen konnten.

Die folgenden kurzen Punkte gehen nicht systematisch auf die einzelnen Beiträge des Colloquiums ein, sondern schließen sich an Themen an, die im Vordergrund der Debatten standen und die - vom skandinavischen Standpunkt aus - für die Problematik von Bedeutung erscheinen.

\footnotetext{
${ }^{4}$ Zusammenfassende Gesichtspunkte und Literaturangaben bei: Nils Runeby, Das ,bedingte' Erbreich: Schweden, in: Der dynastische Fürstenstaat, hrsg. von Jobannes Kunisch (Berlin 1982). Es ist auffallend, wie vieles in der frühen niederländischen Debatte, Auseinandersetzungen über verschiedene ,Mischformen', Fragen nach Widerstandsrecht, Ephoren, Majestas realis etc., theoretisch gesehen, sich auch in einen schwedischen Zusammenhang einfügen ließe. „Die junge Republik“, schreibt Dr. Mout, „mußte bis in die fünfziger Jahre des siebzehnten Jahrhunderts ohne eine ausgeglichene Struktur politischer Theorien von ausgesprochener republikanischer Prägung auskommen“. Erst dann wurde Holland gefährlich" (vgl. oben).

${ }^{5}$ Robert Oresko kann anhand der von ihm analysierten, sehr komplexen Situation feststellen, daß in Genf "as with so much in early modern Europe the distribution of power“ „illdefined and fluid" war. "It is embarrassing to note at a colloque devoted to republicanism that although the segneurie of Geneva not infrequently referred to the republic of Geneva the concept of republicanism in the city had not developed to a notably sophisticated level“. „Evidence for a fully developed consciousness of the distinctions between republican and princely forms of government is thin."

${ }^{6}$ Vgl. Michael F. Metcalf, Structuring Parliamentary Politics: Party Organization in Eighteenth Century Sweden, in: Parliaments, Estates and Representation 1 (1981).
} 


\section{II}

Im Zusammenhang mit ,richtigen' Republiken, Halbrepubliken, potentiellen Republiken und verhinderten Republiken sind mehrere Fragen gestellt worden, u. a., inwiefern die geographische Lage, die Frage von Zentrum und Peripherie für Staatsformen in Europa von Bedeutung gewesen sein könnte. Eine Lage an der Peripherie dürfte wohl bessere Voraussetzungen für eine, abweichende' Staatsform geboten haben (das Alpengebiet, Skandinavien, Polen). Natürlich muß auch die Problematik, inwieweit die Größe des Territoriums eine Rolle spielte, beachtet werden. Es ist ein Unterschied, ob man mit Städten, Kommunen, Provinzen oder größeren Flächenstaaten arbeitet. Wichtig muß weiter die Chronologie sein. Es ist ein Unterschied, ob man sich im 15. oder im 17. Jahrhundert befindet.

Auf der anderen Seite muß man auch mit sehr zähen Strukturen rechnen, die lange, vor allem auf der Lokalebene, überleben konnten, zumal wenn eine Zentralmacht fehlte, schwach war oder geographisch weit entfernt lag. Dies geht deutlich aus den Beiträgen hervor, ob es sich nun um Beispiele aus Italien im Mittelalter oder aus dem revolutionären England im 17. Jahrhundert handelt. Mehr oder wenig selbständige, ,feodale' Eliten haben sich lange erhalten oder sich rechtzeitig erneuern können. "Zentrum" und „Peripherie“" stellen auch eine innenpolitische Problematik dar. Die schwedischen und finnischen ,Randgebiete" waren für die Zentralregierung mit ihren ,zivilisatorischen' Ambitionen offenbar nicht leicht $\mathrm{zu}$ erreichen ${ }^{7}$. Die Problematik kann vielleicht anhand eines etwas extremen Beispiels verdeutlicht werden, eines Landes nämlich, das sowohl an der wirklichen Peripherie Europas liegt als auch ein fest umrissenes Gebiet darstellt: Island. Island war Teil des dänischen Staates, lag aber natürlich von Kopenhagen weit entfernt. Die Möglichkeit der Zentralregierung und der absoluten dänischen Könige (nach 1660), sich auf Island durchzusetzen, hatte seine Grenzen.

In einer neuen Darstellung über Island im 18. Jahrhundert wird festgestellt, daß es vor allem die isländischen Beamten waren, „who could influence decision-making in Icelandic issues in the 18 th century Danish monarchy“. Die Bauern hatten keine Möglichkeit, direkten Einfluß auszuüben, und die Beamten ließen keine Auffassungen, die sich gegen ihre Interessen gerichtet hätten, bis Kopenhagen durchdringen. Die Beamten ihrerseits vertraten die Interessen der lokalen Großgrundbesitzer. „There is good reason to speak about the Icelandic elite.“ Das absolutistische Regiment „upheld the traditional social structure, and the Icelandic elite could continue to govern their country in the name of the king“. Diese Bereitschaft der Zentralregierung, schreibt der Verfasser Harald Gustafsson, mit einer lokalen Elite Kompromisse zu schließen, „is typical for the policy of the Danish absolutist regime, also in other parts of the monarchy. In my opinion, this behaviour is also characteristic for the limits of the exercise

7 Zur Frage von Zentrum und Peripherie in Schweden/Finnland siehe die wichtige Aufsatzsammlung von Matti Klinge, Runebergs två fosterland (Borgå 1983). Vgl. weiter Nils Runeby, Barbarei oder Zivilităt. Zur Entwicklung einer organisierten Gesellschaft in Schweden im 17. Jahrhundert, in: Europe and Scandinavia (Anm. 3). 
of power in early modern Europe, not the least in the ,absolutist' monarchies." Versuche, die Stellung der Bauern im Verhältnis zu den Großgrundbesitzern zu stärken, wurde nie zu Ende geführt, weil man keine Möglichkeit sah, die Reform in der Praxis durchzusetzen ${ }^{8}$.

In welches Fach man nun die isländischen Zustände einordnet, scheint mir eine ganz offene Frage zu sein. Es spielte für die Isländer keine große Rolle, ob die Regierung in Kopenhagen aristokratisch oder monarchisch war. Dr. Roys Urteil, „that continuity of membership of the ruling body, long service to the city and a kind of Aldermanic solidarity stood in the way of any hasty or dramatic changes at the top", bezieht sich zwar auf englische Städte, scheint aber auch auf isländische Verhältnisse anwendbar zu sein. Wichtig ist auch der Hinweis Dr. Blockmans (für eine andere Periode), daß in der Praxis der Städte eine alternative Kultur und eine eigene Tradition sich herausbildeten ${ }^{9}$. Es scheint mir eine zentrale Frage zu sein, der Entwicklung von der feudalen vicinia zu einer, modernen“ Bürokratie nachzugehen.

\section{III}

Es ist hier auch von Bedeutung, nach dem Selbstverständnis der Eliten (auf verschiedenen Ebenen) zu fragen. Klar ist die Problematik von Dr. Bertelli formuliert worden, nämlich, ob man von einer gemeinsamen "Weltanschauung" der Oligarchien während einer gewissen Zeit sprechen könne. Die Frage ist „de decouvrir si, pour chaque période oligarchique on peut tracer certaines lignes fondamentales (religieuses, morales) communes aux groupes dominants“. Während der von ihm behandelten Periode hebt Bertelli die Aufrechterhaltung des sozialen Friedens, als commune bonum aufgefaßt, vor allem in Zeiten von Standesunruhen oder Auseinandersetzungen zwischen verschiedenen Parteien, hervor. Verbunden damit sind "des normes de ,civilité, de ,bonnne conduite', qui servent d'exemple aux classes subalternes, mais qui distinguent aussi les couches supérieures des couches inférieures“. Die Stadtoligarchien der

${ }^{8}$ Harald Gustafsson, Mellan kung och allmoge - ämbetsmän, beslutsprocess och inflytande på 1700-talets Island (Stockholm Studies in History 33, Stockholm 1985). Siehe auch ders., Upplysning, kameralism och förvaltning - en aspekt på det danska styret av Island på 1700-talet, in: 1700-tals studier tillägnade Birgitta Ericsson (Stockholm Studies in History 34, Stockholm 1985). Zur Entwicklung auf Island im 17. Jahrhundert vgl. Lýdur Björnsson, Island i det oldenborgske rige i det 17. århundrede, in: Magtstaten i Norden i 1600- tallet og de sociale konsekvenser (XIX nordiske historikerkongres Odense 1984, 1, Odense University Studies in History and Social Sciences 85, Odense 1984).

${ }^{9}$ Ein zunehmender Einfluß der Elite im Laufe des 18. Jahrhunderts ist für die finnischen Städte nachgewiesen worden: „Zu beachten ist ferner, daß seit den 1730er Jahren gerade die ärmere Bevölkerung immer häufiger ihre Bürgerrechte verlor. - Die Entwicklung der Stadtverwaltung im 18. Jahrhundert war somit charakterisiert durch eine Plutokratisierung und eine stark elitistische Strömung" (Ilkka Mäntylä, Valitut, ehdollepannut ja nimetetyt. Pormestarien vaalit 20 kaupungissa 1720-1808 [Helsinki 1981) 209). Zur Entwicklung der städtischen Administration in Skandinavien siehe Birgitta Ericsson u.a., Stadsadministrationen i Norden på 1700-talet (Oslo/Bergen/Tromsø 1982). 
Renaissance wollten mitten in den Unruhen „un modèle d'honnête homme gentil“ geben $^{10}$.

Formuliert auf diese Weise, scheinen die Vorstellungen merkwürdig bekannt zu sein. Es gab in Schweden im 16. und 17. Jahrhundert eine klar feststellbare, adelige Elite, eng durch Heirat und Besitz zusammengehalten, die die führenden Ämter innehatte, im Reichsrat vertreten war, und die sowohl eine politische als auch eine ökonomische Elite darstellte. Die Familien hatten auch ihre Klienten und bildeten Clans im älteren Stil. Noch klarer sind die Verhāltnisse im dänischen Reich, das - wie erwähnt - in Schweden als aristokratisch dargestellt werden konnte. Dänemark, schreibt Erling Ladewig Petersen, unterscheidet sich sowohl vom europäischen Kontinent als auch von dem „schwedischen Ständestaat" durch sein „elitär aristokratisches Staats- und Stāndesystem", und die Staatsform ist weder als eine Monarchie noch als eine Republik, sondern als eine „Dyarchie“ (Knud Fabricius) beschrieben worden, um das Verhältnis zwischen König und Rat zu charakterisieren ${ }^{11}$.

Das adelige Selbstverständnis erhielt nun in Schweden seinen symbolischen Ausdruck in dem sog. Ritterhaus in Stockholm. Hier tagte der Adel während der Reichstage, das Haus beherbergte auch den Adelsstand als Korporation, und hier mußten sich die adeligen Familien, um als Adel anerkannt zu werden, eintragen lassen ${ }^{12}$. Das Stockholmer Ritterhaus hatte eine reprāsentative Funktion und kann somit mit den europäischen Ratshäusern verglichen werden.

Der Bedarf, ein Haus zu bauen, lebte während der Vormundschaftsperiode in den 1630er Jahren auf. Axel Oxenstierna erklärte im Jahre 1638, er wolle ein „palatium Italicum“ haben, und der Gouverneur von Stockholm wünschte sich im Jahre 1642 „ein großartiges / magnifique / Haus“, „so daß wir damit Ruhm erwerben können“. Das Haus wurde - mit den Worten des Kunsthistorikers Allan Ellenius - „zu einem Stück ,architecture parlante', das in der schwedischen Architekturgeschichte kaum seinesgleichen hat". „Hier nimmt nun Ideologie sichtbare Gestalt an, so als wäre man plötzlich in einen Hörsaal der Universität Uppsala versetzt, wo ein junger Adeliger eine Rede über den Wert der Tugend hält ...“.

In dem Ritterhaus ist in Skulpturen, Malereien und Inschriften der ganze herkömmliche Tugendkatalog vorhanden. Hier kommt „jenes pädagogische Konzept“ zum Vorschein, das eine Verbindung „von heroischer Tugend mit gelehrten Studien zur Ausbildung des vollkommenen Adels" darstellt: Nobilitas, Valor, Honor, Prudentia, Fortitudo, Studium. Die Liebe zum Vaterland wird hervorgehoben, das Beispiel

${ }^{10} \mathrm{Zu}$ den entsprechenden ,Zivilitāts- und ,Ordnungs-Vorstellungen des 17 . Jahrhunderts in Schweden vgl. Runeby (= Anm. 7).

${ }^{11}$ Ladewig Petersen (= Anm. 2).

${ }^{12}$ Für die folgende Darstellung siehe Allan Elleniws, Die repräsentative Funktion adeliger Bauten und ihre Ausstattung im schwedischen 17. Jahrhundert, in: Arte et Marte. Studien zur Adelskultur des Barockzeitalters in Schweden, Dānemark und Schleswig-Holstein, hrsg. von Dieter Lobmeier (Neumünster 1978) sowie den Anhang des Buches: Das ikonographische Programm des Ritterhauses in Stockholm. Vgl. weiter Margareta Revera, En barock historia. Om den svenska 1600-talslyxen och dess plats i samhällsomvandlingen, in: Tre Karlar. Karl X Gustav, Karl XI, Karl XII (Stockholm 1984) mit Literaturangaben. 
der Vorfahren sorgt für Tradition und Legitimität, und es wird auch betont, daß „die Stärke der Staatsbürger die beste Stütze des Reiches /sei/“. In den adeligen Stadtpalästen und Landsitzen findet man auch diese Zusammenstellung von klassischem $\mathrm{Hu}$ manismus und protestantischem Glauben.

Als der Maler David Klöcker in den 60er Jahren anfing, den großen Plafond des Ritterhauses zu gestalten, fügte er Adel und Reich unauflöslich zusammen, und - nach Klöckers eigener Beschreibung - ,in der Mitten vor dem Throne, ist Concordia, die Eintracht, mit beyden Armen augestrecket, in action, als wenn sie alle die gleich gemeldeten Tugenden in ihre Arme fassen und dem Königreiche einverleiben wolte“. Svecia von Concordia beschützt war ein Programm ${ }^{13}$. Das „hocharistokratische“ Regiment in Schweden weckte, hebt Sven A. Nilsson hervor, „antagonistische Kräfte“, die in hohem Grade die Vormundschaftsperiode charakterisierten. In dieser Situation wurde Concordia zu einer Zentralfigur. Reichsregierung, Reichsrat und ,tugendhafter' Adel wurden zu einer Garantie der notwendigen Eintracht des Reiches ${ }^{14}$. Natürlich ist die Symbolik des Ritterhauses Allgemeingut, aber die Ambitionen des Hochadels als Ausdruck für „die geistige Physiognomie der führenden Familien“ sind trotz allem bemerkenswert. In der gemischten Monarchie wurde der Anspruch erhoben, Teil an der Machtausübung und Verantwortung für das Ganze zu haben, Gegenspieler des Königs und die beste Stütze des Reiches zu sein. Das Ephoren-Argument steht hier im Zentrum ${ }^{15}$.

In dem Ausmaß, wie sich die Elite an der Regierung des Reiches beteiligt sah, war sie natürlich auch an dem Schaffen einer nationalen Identität und einer nationalen Mythologie (z. B. der ,gotischen' Vergangenheit Schwedens) beteiligt ${ }^{16}$. Gustav Adolf, der die ,gotische' Tradition ausnutzte, ließ es sich angelegen sein, das Alter und die Größe des schwedischen Reiches hervorzuheben. Auch war es für ihn wichtig, daß Schweden nie dem Kaiser untergeordnet gewesen war und somit als ihm ebenbürtig dastand. Man muß sich hier nur bewußt machen, daß z. B. König, Rat und Aristokratie durchaus unterschiedlicher Auffassung darüber sein konnten, welche Tradition die wahre war, und wie die Geschichte interpretiert werden sollte. Diese Frage mag hier weniger das Verhältnis zum Kaiser oder die gotische‘ Vergangenheit betreffen, als vielmehr sich darauf beziehen, wie z. B. die Fundamentalgesetze und deren Gültigkeit interpretiert werden sollten. Natürlich kann in diesem Zusammenhang von seiten des Rates oder von seiten einer der Stände die Rede auf eine Einschränkung resp. Kon-

${ }^{13}$ Allan Ellenius, Karolinska bildidéer (Ars Suetica 1, Uppsala 1966) $45 \mathrm{ff}$.

${ }_{14}$ Sven A. Nilsson, Den karolinska militärstaten. Frederis problem och krigets, in: Tre Karlar (= Anm. 12).

15 In dieser Situation kann man den Schlußfolgerungen von Dr. Fröschl nur zustimmen, daß nämlich die Darstellungen in den Rathäusern sich ,als exempla virtutis in den gesamteuropäischen kulturellen Zusammenhang“ einfügen lassen. Man erkennt hier die „fundamentale Bedeutung" "einer, die politisch-kulturellen Eliten sowohl der Monarchien wie der nichtmonarchischen Staatswesen verbindenden und umfassenden Bildungstradition".

${ }^{16}$ Die wichtigste Darstellung über die gotische‘ Tradition in Schweden ist immer noch Joban Nordström, De Yverbornes ö. Sextonhundratalsstudier (Stockholm 1934). Siehe auch Barudio (= Anm. 2). 
trolle der Königsmacht gekommen sein, auf eine Verteidigung des Reiches und dessen ,wahre" historische Kontinuität einem widerspenstigen König gegenüber ${ }^{17}$.

Es ist auch wichtig hervorzuheben, daß die adelige Elite im Laufe des 17. Jahrhunderts in die neue Bürokratie hineinwuchs, wobei gleichzeitig eine umfassende Nobilitierung stattfand, um Personen für die neue Verwaltung zu rekrutieren. (In dieser Beziehung war Schweden ,offen nach unten ${ }^{18}$.) Im Zusammenhang sowohl mit dem Absolutismus und der sog. Freiheitszeit als auch im Vergleich z. B. mit Dänemark während des ,bürokratischen Absolutismus' ist es notwendig, diese Kontinuität zu betonen. Der König war sowohl im absolutistischen Dänemark als auch im Schweden ,der herrschenden Stānde' zeitweise nur ein Spielball ${ }^{19}$.

\section{IV}

Ein weiterer Hinweis zur Ephoren-Problematik muß hier angefügt werden. Es ist seit langem nachgewiesen, daß in Schweden im 17. Jahrhundert das Ephoren-Argument anzutreffen ist und daß der Reichsrat als „ephori regni“, als Mittler zwischen König und Volk, bezeichnet wurde. Eine Komplikation muß aber besonders hervorgehoben werden. An der Spitze des Reichsrates befanden sich die fünf hohen Reichsbeamten, die je ein zentrales Collegium unter sich hatten. Der Reichsdrost konnte sich sogar als Vize-König bezeichnen. Durch die vielen Kriege und durch die Vormundschaftsregierungen wurde das Fünfergremium besonders bedeutungsvoll.

${ }^{17}$ Etwas von dieser Problematik steckt auch in der heiklen Frage, wer Zugang zum und Verfügungsrecht über das Archiv des Reiches, d.h. über dessen Geschichte, haben sollte. Siehe Sven Lundkvist, Riksens arkiv och makten. En studie i riksarkivets utveckling före 1618, in: Arkivvetenskapliga studier 5 (Stockholm 1981). Zu den schwedischen Fundamentalgesetzen vgl. zusammenfassend Nils Runeby, Till frågan om fundamentallagsbegreppets uppkomst i Sverige, in: Den svenska juridikens uppblomstring i 1600-talets politiska, kulturella och religiösa stormaktssamhälle, hrsg. von Göran Inger (Rāttshistoriska studier 9, Stockholm 1984) mit Literaturangaben.

18 „In Wirklichkeit gab es keine Hindernisse für einen wirklich hervorragenden Nichtadeligen, die höchsten Verwaltungsposten zu erreichen. Bevor er den Punkt in der Karriere erreicht hatte, wo Adelsstand notwendig war, wurde er für seine Verdienste geadelt. Der schwedische Adel war kein geschlossener Stand, sondern erneuerte sich immer wieder durch Nobilitierungen" (AnnaBrita Lövgren, Stormaktstidens förvaltning, in: Tre Karlar (= Anm. 12)).

${ }^{19}$ Der Charakter des schwedischen Staates und der schwedischen Gesellschaft während der Freiheitszeit ist verschiedentlich interpretiert worden. Gunnar Artéus hat in einer umfassenden Untersuchung die These aufgestellt, daß Schweden eine „militarisierte“ Gesellschaft gewesen sei. Nach seiner Definition „a militarized society is one, the political, social, economic and cultural structure of which appears to have been shaped essentially by military ideas or interests. It could be argued that Sweden of the Liberty era was a society of this kind."Während der Zeit von 1682 bis 1812 ,the armed forces did away with three constitutionally established systems of government, Carolean absolutism, the parliamentary rule of the Liberty era and the Gustavian autocracy“, die alle eine Drohung „to their vital interests as a corporation“ waren, aber sie haben nicht den Ehrgeiz gehabt, selbst „, military regime“ zu bilden. Der Durchschnittsoffizier „was more of a country squire (lantjunkare) than a soldier and he was hostile to dictatorships - of any kind“ (Krigsmakt och samhälle i frihetstidens Sverige [Stockholm 1982]). Vgl. die Debatte bei Michael F. Metcalf, Den militariserade frihetstiden?, in: Historisk tidskrift [Schweden] (1985) 1 und Gunnar Artéus, Om det frihetstida samhällets karaktär, in: Historisk tidskrift [Schweden] (1985) 3. 
Die Aufgabenbereiche der Reichsbeamten, die eng mit der Entwicklung der Zentralverwaltung zusammenhingen, wurden nach dem Tode Gustav Adolfs durch die sog. Regierungs-Ordnung von 1634 festgelegt. Die Regierungs-Ordnung knüpft an die ganze Verwaltungsarbeit während der Zeit Gustav Adolfs an. König und Kanzler hatten das gemeinsame Ziel, eine Staatsmacht aufzubauen, die stark genug sein sollte, um das schwedische ,Militärsystem ' der neuen schwedischen Großmacht zu tragen. Nach dem Tod des Königs und im Zuge der Regierungs-Ordnung gab es - in der Interpretation Sven A. Nilssons - keinen Platz mehr für das persönliche Regiment des Königs. Oxenstierna baute das System weiter aus. Er schuf eine feste, selbständig arbeitende Verwaltung unter der Führung der aristokratischen Elite mit den Reichsbeamten an der Spitze, eine zentralisierte Verwaltung, die auch einige Aufgaben des Reichstages übernahm.

„Die Regierungs-Ordnung“, schreibt Sven A. Nilsson, „schafft eine Alternative zu dem von einem König geführten Staat, eine Staatsmacht in den Händen von den hohen Reichsbeamten, stark genug, ein Land in einem großen Krieg regieren und die Schwierigkeiten einer Vormundschaftzeit überwinden zu können.“ Die RegierungsOrdnung ist auch als „eine Barriere gegen zukünftige, ungeeignete Monarchen“ gedacht. „Es sind alte, räterepublikanische Gedanken, die hier zum Vorschein kommen.“20 Die Regierungs-Ordnung bezeichnet sich auch als „ewig“. Das neue System „machte zwar nicht immer die Beamten zu Anhängern des aristokratischen Regiments. Aber sie wurden Diener des Staates und der Staatsmacht." ${ }^{21}$ Die RegierungsOrdnung ist von den folgenden Königen nicht anerkannt und die Ephorenterminologie während des Absolutismus ausdrücklich verboten worden. Man könnte aber die Frage zur Diskussion stellen, ob nicht das hier geschaffene Verwaltungssystem, das ohne König arbeiten konnte, auch eine Voraussetzung für die fast republikanische Entwicklung Schwedens im 18. Jahrhundert war.

Auf der anderen Seite wird mit der Annahme des neuen ,Systems“ (Militärstaat, Machtstaat) eine zwingende Kontinuität zwischen gemischter Monarchie, Absolutismus und Freiheitszeit herausgestellt, und die Frage nach der Staatsform wird fast sekundär ${ }^{22}$. Im Zentrum stehen stattdessen Zentralisierung, Ubberwachung und Kon-

${ }^{20}$ Die Regierungs-Ordnung ist in der schwedischen Historiographie lebhaft diskutiert worden. Siehe zusammenfassend Sven $A$. Nilsson, 1634 års regeringsform i det svenska statssystement, in: Statsvetenskaplig tidskrift 1984, mit Literaturangaben.

${ }^{21}$ Sven A. Nilsson (= Anm. 14) 35.

${ }^{22}$ Natürlich müssen hier gewisse Einschränkungen gemacht werden. „Naturally, the Swedish council did not function in exactly the same manner throughout the 17 th century. Its working procedure depended to some extent on whether the monarch was of age or the Council served as a regency government. In other respects, the working procedure was determined by the increasing quantity of tasks and the emergence of the Caroline absolutism. - Certain measures taken to increase efficiency - in Sweden and in other countries - were obviously only possible due to the powerful position of the king" (Anna-Brita Lövgren, The King's Council in Sweden and in Europe during the 17th Century, in: Europe and Scandinavia (= Anm. 3). Die Veranderung wurde nach Auffassung Lövgrens hauptsächlich durch Wünsche nach Spezialisierung und Effizienz hervorgerufen; die Verbindung zwischen Verwaltung und Regierung blieb bestehen. Siehe auch Lövgren (= Anm. 18). Zu den Begriffen ,Machtstaat' und ,Militärstaat' siehe Ladewig Petersen (= Anm. 2) und Magtstaten i Norden (= Anm. 8). 
trolle und eine Expansion der Verwaltung. Die Entwicklung war von der Notwendigkeit geprägt, die ökonomischen Möglichkeiten so weit wie möglich auszuschöpfen, und sie hatte natürlich auf der lokalen Ebene Folgen für die herkömmliche Selbstverwaltung, für den vorhandenen Kommunalismus. Die neuen ,Landeshauptmänner (so wie die Geistlichkeit) wurden in den staatlichen Kontrollapparat eingefügt und sollten lokal die Interessen der Regierung und der Zentralmacht wahrnehmen. Die Organisation, die zentral unter Umständen als Alternative zur Königsmacht hätte wirken können, konnte lokal zu einem Abbau der traditionellen Verwaltungsformen und zu einer Zerstörung des alten Kommunalismus führen.

Im 17. Jahrhundert kann auch eine beachtliche Anzahl von Stadtgründungen verzeichnet werden. Sie sind als Teil einer staatlichen Lokalisierungspolitik gesehen worden mit der Absicht, die zentrale Kontrolle über ökonomisch wichtige Gebiete und abseits gelegene Teile des Reiches zu stārken. Die Stādte wurden als Handels-, aber auch als Verwaltungs-, Steuer- und Militārzentren aufgebaut ${ }^{23}$, und königliche Bürgermeister wurden sowohl in den neuen als auch in den alten Städten ernannt. Daß die ,gelehrten' Bürgermeister aber von adeliger Seite angeklagt werden konnten, in ihren Ansprüchen ,republikanische“, ,holländische“ Ansichten zu vertreten, geht aus dem oben angeführten Pamphlet hervor.

Die Zentralisierung traf natürlich auch die ländlichen Gebiete. Die alten Thingsgemeinden verloren an Bedeutung, die lokale Rechtssprechung wurde in ein System mit einer festen Appellationsordnung eingefügt, und das römische Recht wurde weitgehend rezipiert ${ }^{24}$. Es war bezeichnend, wenn die Regierung vor dem Reichstag 1635 (nach der Annahme der Regierungs-Ordnung) in einem Brief an Bischöfe und Landeshauptmänner unterstrich, daß diese mit Hilfe der Geistlichkeit zusehen mögen, daß zum Reichstag „keine aufständischen Bauern aus promiscua plebe kommen können, sondern wohlmeinende, redliche Männer, die von einiger Discretion sind und das Wohl des Vaterlandes wünschen“. Auf diese Weise wollte man allen unangenehmen. Úberraschungen aus der Provinz vorbeugen.

Es muß auch betont werden, daß die Geistlichen - trotz Widerstand - herangezogen wurden, um eine kontinuierliche Volkszählung als Grundlage für die Steuererhebung und die Konskription durchzuführen ${ }^{25}$. In diesem bestimmten Sinne wurde die schwedische Geistlichkeit ,bürokratisiert'; nicht Selbständigkeit, sondern Loyalität wurde gefordert. Am Ende des Jahrhunderts wurden sie auch beauftragt, zuzusehen, daß die Bevölkerung einigermaßen ,lesen' lerne. Der Staatsbürger mußte, um die Vorschriften der Kirche und des Staates richtig aufzufassen und um sich ,Lebenskompe-

${ }^{23}$ Birgitta Ericsson, De anlagda städerna i Sverige (ca. 1580-1800), in: De anlagte steder på 16001700 tallet (Urbaniseringsprosessen i Norden 2, XVII nordiske historikermøte Trondheim 1977, Oslo/Bergen/Tromse 1977). Ein reich differenziertes Bild gibt das in Anm. 9 erwähnte Sammelwerk.

${ }^{24} \mathrm{Zu}$ der Entwicklung des Rechts vgl. die Aufsatzsammlung Den svenska juridikens uppblomstring (= Anm. 17)

${ }^{25}$ Sven $A$. Nilsson, Krig och folkbokföring under svenskt 1600-tal, in: Scandia (1982). 
tenz' (E. Johansson) in der lutherischen Weltordnung und in der neuen Gesellschaft $z u$ verschaffen, lesen können ${ }^{26}$.

Die letztgenannte Aufgabe zeigt die Komplexität der Situation. Die Bauern wurden zwar überwacht, blieben aber als Stand im Reichstag. Es gab auch innerhalb des Systems Kanäle, um ihre Interessen an die Öffentlichkeit zu bringen, und sie konnten auch auf lange Sicht sich ökonomisch in einer Situation behaupten, wo die adelige Ökonomie schwankte und die Stellung der hochadeligen Magnaten zu Gunsten der kleinadeligen und nichtadeligen Bürokratie untergraben wurde ${ }^{27}$. Man kann in einer längeren Perspektive sich natürlich auch darüber Gedanken machen, was die Kunst des Lesens für das Selbstbewußtsein der Bevölkerung bedeutet hat ${ }^{28}$.

In den 1830er Jahren richteten die schwedischen Liberalen, zum Ärger des ehemaligen Revolutionsgenerals Jean Baptiste Bernadotte, heftige Kritik gegen die bestehende Monarchie. Der führende liberale Journalist Lars Johan Hierta äußerte sich 1834 über die richtige Staatsform: „Es muss zugegeben werden, dass in der konstitutionellen Monarchie selbst und in der repräsentativen Gesellschaftsordnung eine republikanische Richtung in der Tat zu finden ist, falls man die politische Selbständigkeit des Volkes und die Einschränkung des Gutdünkens durch Gesetz als Frucht der republikanischen Lehren betrachtet ..." Hierta findet auch eine brauchbare Formel, um die richtige ,Mischung' zu beschreiben: „eine Monarcbie mit republikaniscben Institutio$n e n^{\iota 29}$. Diese Beschreibung ließe sich auch auf frühere Perioden der schwedischen Ge-

${ }^{26}$ Egil Jobansson, Den kyrkliga lästraditionen i Sverige - en konturteckning, in: Ur nordisk kulturhistoria 3 (XVIII nordiska historikermötet, Studia historica Jyväskylāensia 22,3, Jyvāskylä 1981). Ich habe diesen Hinweis schon früher in dem oben Anm. 7 erwāhnten Aufsatz gemacht.

${ }^{27}$ Zur Entwicklung des Bauernstandes aus verschiedenen, aber inhaltlich nicht unvereinbaren Ansatzpunkten siehe Jan Lindegren, Den svenska militärstaten 1560-1720, in: Magtstaten i Norden (= Anm. 8) und Margareta Revera, 1600-talsbönderna och deras herrar. Om jordägande, skatter och samhällsförändring i ljuset av nyare forskning, in: Den svenska juridikens uppblomstring (= Anm. 17). Revera kann sich hier, was die ,selbständigen' Bauern betrifft, mit großer Deutlichkeit ausdrücken. Es ist „apparent that seventeenth century freeholders comprised a comparatively favored category of peasants in the political and social as well as the economic sense. Their representation in the Riksdag appears to have been strong, their land ownership was respected in those situations where it had practical importance, and they were not subjected to unlawful increases in taxes under the nobility." Am meisten betroffen scheinen die Kronbauern gewesen zu sein.

${ }^{28}$ Natürlich gab es auch Vertreter ganz anderer Strömungen, die die neue Staatlichkeit ablehnten und neue Lösungen und Freiheiten suchten. Zum, utopischen' Denken in Schweden siehe Ronny Ambjömsson, Den sista striden. Om en svensk pacifist från 1600-talet, in: Lychnos (19791980). Auch gedruckt in ders., Det okända landet. Tre studier om svenska utopister (Stockholm 1981).

${ }^{29}$ Zitiert nach Jean Göransson, Aftonbladet som politisk tidning 1830-1835 (Uppsala 1937) $98 \mathrm{f}$. Zur Republikdebatte in Schweden in den 1830er Jahren vgl. Nils Runeby, Den nya världen och den gamla. Amerikabild och emigrationsuppfattning i Sverige 1820-1860 (Studia historica upsaliensia 30, Uppsala 1969) Kap. 5. 
schichte anwenden, sie klingt fast wie eine leicht ,modernisierte Variante der Worte Axel Oxenstiernas an Whitelocke. Hierta schrieb diesen Artikel auf den Tag 200 Jahre nach der Regierungs-Ordnung. Ob sich nun hinter der Fassade ein Machtstaat oder eine wahre Gemeinschaft, wo alle zufrieden sind und „indeavour nothing of disquiet unto either", verbirgt, mag in der schwedischen Monarchie noch immer eine Diskussionsfrage sein. 